\title{
Co-operative Spring
}

\begin{tabular}{|c|c|c|c|c|c|c|c|}
\hline $\begin{array}{l}\text { Compiled by } \\
\text { DOROTHY WADE } \\
\text { Regina }\end{array}$ & 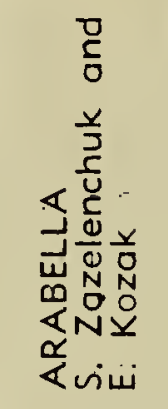 & 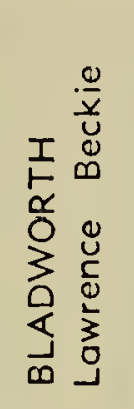 & 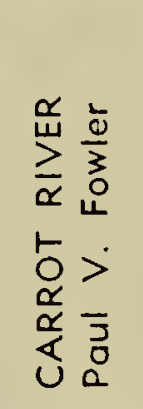 & 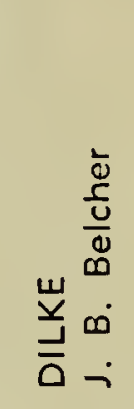 & 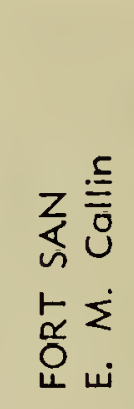 & 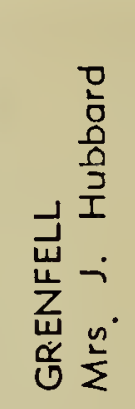 & 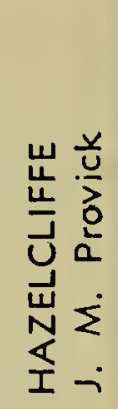 \\
\hline Whistling Swan & & Ap15 & My 2 & & Ap 9 & Ap23 & Ap22 \\
\hline Canoda Goose ...................... & Mr29 & Mr28 & Ap 5 & Mr28 & Mr23 & Apl 4 & Mr28 \\
\hline Mallard & $M r 28$ & Mr28 & Ap 3 & & Mr28 & Ap 1 & Ap 7 \\
\hline Pintail _.......... & Ap 6 & Mr28 & Ap 3 & $\mathrm{Mr} 28$ & $\mathrm{Mr} 24$ & Mr31 & Mr31 \\
\hline Morsh Howk & Ap 1 & Mr21 & Ap 9 & Mr22 & $\mathrm{Mr} 24$ & Ap 5 & Mr25 \\
\hline Killdeer _....___. & Mr30 & Mr30 & Aplo & $\mathrm{Mr} 27$ & Ap 5 & Ap20 & Mr28 \\
\hline Common Snipe & Ap25 & My 4 & MY 1 & & & & \\
\hline Mourning Dove _................. & My10 & Myl6 & My 1 & My 9 & Ap23 & Ap21 & Apl6 \\
\hline Common Nighthowk & My27 & My25 & $\ln 3$ & My26 & & In 1. & \\
\hline Ruby-throated Hummingbird - & My29 & & & & In 1 & My26 & $M \vee 26$ \\
\hline Yellow-shafted Flicker ........... & Aplo & Apl3 & Apio & Apl 8 & Ap 13 & Ap 10 & Ap 7 \\
\hline Eastern Kingbird & & Myi6 & $\ln 2$ & My15 & My18 & My17 & Ap 7 \\
\hline Eastern Phoebe & Ap 23 & & & & & My 5 & Ap29 \\
\hline Barn Swallow - & My 5 & My 8 & Myl4 & My 5 & My 3 & My 7 & My 4 \\
\hline Purple Martin & & & & & & My16 & \\
\hline Common Crow & Mr25 & Mr21 & Mr20 & Mrlo & Mrl6 & Mr15 & Mr22 \\
\hline House Wren & My22 & My 29 & My 15 & & Myl1 & My27 & \\
\hline Catbird & & & Myl7 & & My 9 & My27 & \\
\hline Brown Thrasher & & My 15 & & MY31 & Myl4 & Myl 8 & My25 \\
\hline Red-eyed Vireo & & & My20 & & & & \\
\hline Tennessee Warbler & & My20 & My14 & & & & \\
\hline Yellow Warbler & & My17 & & My15 & My10 & My 8 & My24 \\
\hline Myrtle Warbler & My 8 & My 4 & My13 & & My 6 & My 8 & \\
\hline Blackpoll Warbler & & & & My22 & $\ln 2$ & My18 & \\
\hline Ovenbird _._. & & & & & $\ln 2$ & & \\
\hline American Redstart & & & & & Jn 2 & & \\
\hline Redwinged Blackbird & Ap30 & Ap 6 & Apl 7 & & Ap 5 & Ap 5 & Ap 8 \\
\hline Boltimore Oriole & & Myl 5 & My 22 & My21 & Myll & My23 & Apl2 \\
\hline \multicolumn{8}{|l|}{ Rose-breasted Grosbeak } \\
\hline American Goldfinch & & My22 & My27 & & & $M y 26$ & My27 \\
\hline Slate-colored Junco & Mr29 & $\mathrm{Mr} 31$ & Apl0 & & Ap 5 & $\mathrm{Mr} 30$ & Ap 2 \\
\hline Chipping Sparrow & & My 5 & Apl7 & & My 1 & My 9 & \\
\hline White-crowned Sparrow ......... & My 6 & Ap27 & My 2 & Ap27 & My 5 & Ap26 & \\
\hline White-throated Sparrow ...--.-. & My 6 & My 4 & & Ap27 & My 4 & Ap26 & \\
\hline
\end{tabular}




\section{Migration Study, 1963}

\begin{tabular}{|c|c|c|c|c|c|c|c|}
\hline $\begin{array}{l}\text { Compiled by } \\
\text { DOROTHY WADE } \\
\text { Regina }\end{array}$ & 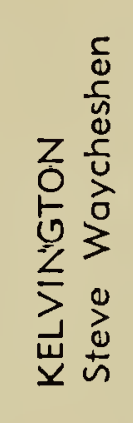 & 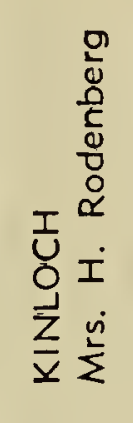 & 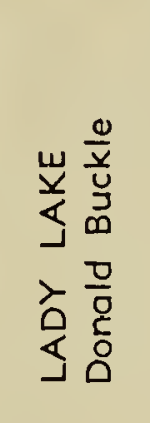 & 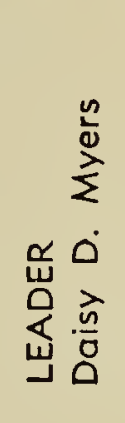 & 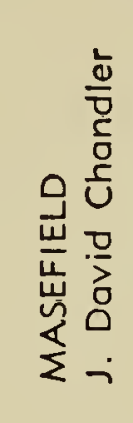 & 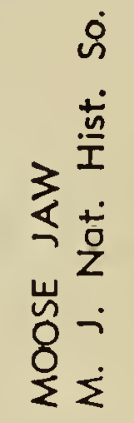 & 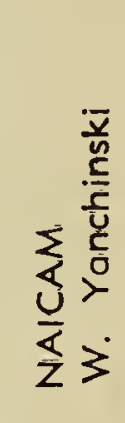 \\
\hline Whistling Swan & & Myl8 & & & & Ap 7 & \\
\hline Canada Goose & Mr28 & $\mathrm{Mr} 31$ & Ap 12 & $\mathrm{Mr} 13$ & $\mathrm{Mr} 22$ & Mr21 & $\overline{A p} 5$ \\
\hline Mallard & Ap 6 & Mr31 & Aplo & & $\mathrm{Mr} 22$ & $M r 22$ & Mr27 \\
\hline Pintail & Ap 6 & Ap 9 & Ap20 & & Mr22 & $\mathrm{Mr} 22$ & Ap 7 \\
\hline Marsh Howk & Mr29 & Apl6 & & & Mr24 & $\mathrm{Mr} 24$ & Mr25 \\
\hline Killdeer & $\mathrm{Mr} 30$ & Ap 9 & Ap 6 & Ap 15 & Mr21 & $M r 31$ & Mr29 \\
\hline Common Snipe & & AD 8 & & & & & My 5 \\
\hline Mourning Dove & Apl 7 & My15 & & Ap 17 & $-M \times 21$ & Apl6 & Ap25 \\
\hline Common Nighthawk & & & & & & My26 & \\
\hline Ruby-throated Hummingbird - & Myl6 & & My29 & & & & \\
\hline Yellow-shafted Flicker ........... & Ap 7 & Ap20 & Ap 15 & Apl7 & & & Aplo \\
\hline Eastern Kingbird .......... & My20 & My24 & Ap 17 & My 9 & & My15 & $\mathrm{My} 24$ \\
\hline Eastern Phoebe & My 1 & Ap20 & & & & & Ap26 \\
\hline Barn Swallow ..._. & My 7 & My 6 & My15 & & & & My 7 \\
\hline Purple Martin & Ap28 & My 6 & & & & My 8 & \\
\hline Common Crow & $\mathrm{Mr} 20$ & Mr25 & $\mathrm{Mr} 25$ & $M: 24$ & $\mathrm{Mr} 23$ & Mrl7 & Mr23 \\
\hline House Wren & Ap28 & My20 & $\overrightarrow{M y l l}$ & & & My21 & My 18 \\
\hline Catbird & My19 & My28 & ? & & & My26 & My30 \\
\hline Brown Thrasher ................. & & & My15 & & & My12 & $\ln 2$ \\
\hline Red-eyed Vireo & My24 & & & & & My26 & My 15 \\
\hline Tennessee Warbler & & & & & & Myl8 & \\
\hline Yellow Warbler ................. & My31 & & & & & Myl1 & Myl6 \\
\hline Myrtle Warbler & & My 8 & My 7 & & & My 1 & $M y 26$ \\
\hline 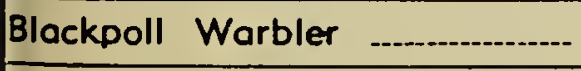 & & & & & & My11 & \\
\hline Ovenbird & My20 & & & & & & \\
\hline American Redstart _............... & My26 & & & & & My20 & Myl7 \\
\hline Redwinged Blackbird & Ap 7 & Ap20 & Ap 10 & & $\mathrm{Mr} 30$ & $\mathrm{Mr} 24$ & Ap 9 \\
\hline Baltimore Oriole & My16 & My21 & & & & Mylo & Myl6 \\
\hline Rose-breasted Grosbeak & Myl5 & My24 & My16 & & $\mathrm{Jn} 10$ & My25 & My20 \\
\hline American Goldfinch & My27 & & Jn 1 & & & Myl4 & $\ln 2$ \\
\hline Slate-colored Junco & $\mathrm{Mr} 24$ & Ap 1 & $\mathrm{Mr} 31$ & Apl6 & $\mathrm{Mr} 30$ & Mr15 & Mr30 \\
\hline Chipping Sparrow & My 5 & Ap 12 & Apl1 & & & My 9 & \\
\hline White-crowned Sparrow & & Ap20 & & & & Ap23 & \\
\hline White-throated Sparrow & My 2 & $A p 20$ & Ap28 & & & Ap28 & Ap 29 \\
\hline
\end{tabular}




\section{Co-operative Spring}

\begin{tabular}{|c|c|c|c|c|c|c|c|}
\hline $\begin{array}{l}\text { Compiled by } \\
\text { DOROTHY WADE } \\
\text { Regina }\end{array}$ & 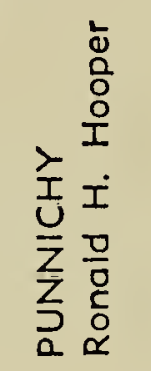 & 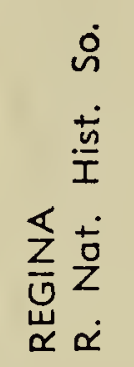 & 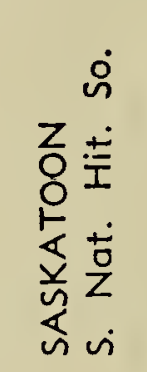 & 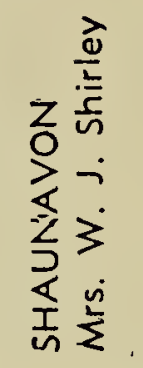 & 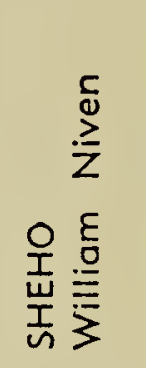 & 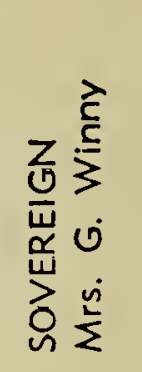 & 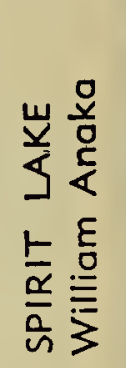 \\
\hline Whistling Swan & & Mr29 & Ap 9 & & Mr25 & Ap 9 & Apl5 \\
\hline Canada Goose & Mr30 & & Mr31 & $\mathrm{Mr} 14$ & Mr21 & Mr27 & Mr22 \\
\hline Mallard & Mr31 & & Mr30 & $\mathrm{Mr} 23$ & Mr28 & $A p 6$ & Mr28 \\
\hline Pintail _............. & Ap 6 & Mris & Mr30 & Mr23 & Mr28 & Ap 8 & Mr31 \\
\hline Marsh Howk & Mr25 & Mr19 & Mr23 & Mr31 & Mr22 & Ap 6 & Mr21 \\
\hline Killdeer & Ap 9 & $\mathrm{Mr} 23^{\circ}$ & $M r 29$ & Ap 9 & $M r 31$ & Ap 10 & Mr31 \\
\hline Common Snipe & Ap21 & Apl 8 & My 4 & My 3 & Ap21 & Ap 18 & Apl 5 \\
\hline Mourning Dove & Ap21 & Apl3 & Apl 2 & Myl4 & Ap 18 & Myl9 & Ap26 \\
\hline Common Nighthowk & & My 26 & My28 & & Myl7 & My26 & My17 \\
\hline Ruby-throoted Hummingbird - & Му31 & & & & My24 & & $\ln 1$ \\
\hline Yellow-shofted Flicker ............ & Apl2 & Ap 5 & Apl3 & & Ap 10 & Ap21 & Ap 7 \\
\hline Eastern Kingbird & My24 & My11 & Myl8 & My23 & My 10 & My25 & My 17 \\
\hline Eastern Phoebe & Ap23 & Apl3 & & & Ap28 & My 8 & $\mathrm{Ap} 13$ \\
\hline Barn Swallow & My 3 & My 1 & My 4 & Myl5 & Ap28 & My20 & Ap 30 \\
\hline Purple Martin & & Ap23 & My 4 & & My 7 & & \\
\hline Common Crow & $M r 24$ & Mrl3 & Mr20 & $\mathrm{Mr} 23$ & Mr22 & Mr23 & Mr21 \\
\hline House Wren & Myll & My11 & My 18 & & Myl1. & My16 & My 10 \\
\hline Cotbird & My11 & My21 & My18 & & My 14 & My 14 & My25 \\
\hline Brown Thrasher & My 17 & Ap28 & My 12 & & Mylo & My18 & \\
\hline Red-eyed Vireo & My28 & My25 & My26 & & My23 & & My24 \\
\hline Tennessee Warbler & & My 5 & My18 & & My 8 & Myl9 & My 15 \\
\hline Yellow Warbler & My10 & My 6 & My 12 & & My 8 & My25 & My19 \\
\hline Myrtle Warbler ....................... & & Apl7 & Ap22 & & My 3 & My 4 & Ap21 \\
\hline Blackpoll Warbler .................. & & My 4 & My18 & & & & \\
\hline Ovenbird & & Myll & My25 & & & & \\
\hline American Redstart & & Myi5 & My18 & & & Myl5 & \\
\hline Redwinged Blackbird & $A p 7$ & Mr29 & Mr30 & Apl3 & $\mathrm{Ap} 6$ & $A p 12$ & Ap 7 \\
\hline Baltimore Oriole & My24 & My 8 & My 12 & & Myl1 & My18 & My16 \\
\hline Rose-breasted Grosbeak & & My12 & My 18 & & My15 & My 8 & My25 \\
\hline Americon Goldfinch & My27 & My15 & My19 & My27 & My19 & My23 & My25 \\
\hline Slate-colored Junco .............. & Mr29 & Mr23 & Mr30 & & $\mathrm{Mr} 27$ & Mr29 & My25 \\
\hline Chipping Sparrow _... & My 4 & Ap27 & My 9 & & My 9 & Ap26 & \\
\hline White-crowned Sparrow & & Ap25 & Ap27 & Ap29 & My 6 & Ap26 & \\
\hline White-throated Sparrow & My 8 & Ap26 & Ap 28 & & My 4 & My 5 & Ap 27 \\
\hline
\end{tabular}




\section{Migration Sfudy, 1963}

\begin{tabular}{|c|c|c|c|c|c|c|c|}
\hline $\begin{array}{l}\text { Compiled by } \\
\text { DOROTHY WADE } \\
\text { Regina }\end{array}$ & 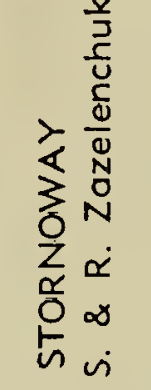 & 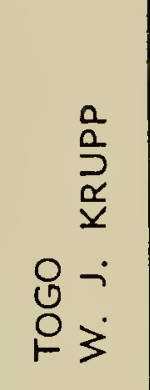 & 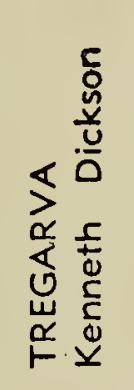 & 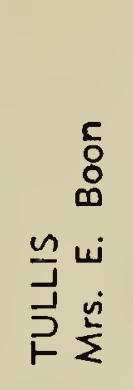 & 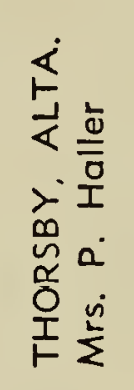 & 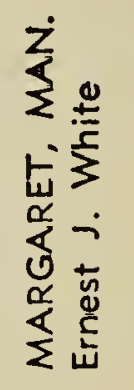 & \\
\hline Whistling Swan & My 1 & & Mr28 & Mr26 & Apl 4 & Ap 8 & \\
\hline Conoda Goose .... & Mr28 & $M+27$ & Mr28 & Mr27 & $\mathrm{Mr} 28$ & Mr3! & \\
\hline Mallard :- & Mr28 & Ap 7 & Mr28 & Mr26 & Mr27 & Mr31 & \\
\hline Pintail - & Mr27 & Ap 13 & Mr29 & Mr26 & Apl3 & Mr28 & \\
\hline Marsh Howk & $\mathrm{Mr} 22$ & Ap 7 & Mr23 & Mr26 & My20 & Mr21 & \\
\hline Killdeer & Ap 7 & A) 6 & Mr22 & Ap 6 & $\mathrm{Mr} 23$ & Mr31 & \\
\hline Common Snipe & Ap29 & & Ap15 & & & & \\
\hline Mourning Dove ..._._. & Apl7 & My 4 & Apl 4 & & & $\mathrm{Ap} \cdot 4$ & \\
\hline Common Nighthawk .................. & & & & & My22 & My29 & \\
\hline Ruby-throated Hummingbird - & & $M y 27$ & & & & My26 & \\
\hline Yellow-shafted Flicker .......... & Ap 9 & Ap22 & AD 6 & & Apl9 & Apl 1 & \\
\hline Eastern Kingbird & & & My14 & My22 & & Myl6 & \\
\hline Eastern Phoebe & Ap 9 & My 5 & My 3 & & & & \\
\hline Barn Swallow & Ap27 & My 5 & My 7 & Myl2 & My21 & MY 9 & \\
\hline Purple Martin & & My 5 & & & & My 3 & \\
\hline Common Crow & Mr22 & Mr20 & Mrl 8 & Mr21 & $\mathrm{Mr} 27$ & Mrl6 & \\
\hline House Wren & & My18 & My25 & & My 8 & My12 & \\
\hline Catbird & My16 & My28 & $M y 26$ & & & My25 & \\
\hline Brown Thrasher & Myl1 & & My18 & & & Myl2 & \\
\hline Red-eyed Vireo & & & My29 & & & & \\
\hline \multicolumn{8}{|l|}{ Tennessee Warbler } \\
\hline Yellow Warbler & & My26. & My 18 & & 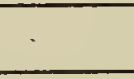 & Myl6 & \\
\hline Myrtle Warbler & My 8 & & My 8 & & & & \\
\hline Blackpoll Warbler & & & Myl1 & & & & \\
\hline \multicolumn{8}{|l|}{ Ovenbird } \\
\hline Americon Redstart & & & & & & $\ln 2$ & \\
\hline Redwinged Blackbird & Ap 1 & Ap 7 & Mr23 & Ap 4 & Mylo & Mr29 & \\
\hline Baltimore Oriole & My 12 & My20 & My25 & & Myl3 & My17 & \\
\hline Rose-breasted Grosbeak & & $\mathrm{My} 26$ & & & My15 & Myl3 & \\
\hline American Goldfinich & & My23 & $\ln 2$ & & & My24 & \\
\hline Slate-colored Junco & $\mathrm{Mr} 31$ & Ap 1 & Mr27 & & Ap 7 & Mr29 & \\
\hline Chipping Sparrow & & Ap 13 & & & & Ap 1 & \\
\hline White-crowned Sparrow & Ap30 & Ap 19 & Ap26 & My15 & My21 & My 2 & \\
\hline White-throated Sparrow & Ap30 & Ap21 & My 8 & & & Ap28 & \\
\hline
\end{tabular}

